\title{
Image Simulations for Strong and Weak Gravitational Lensing
}

\author{
Andrés A. Plazas \\ Department of Astrophysical Sciences, Peyton Hall, Princeton University, Princeton, NJ 08544, USA; \\ aplazas@astro.princeton.edu
}

Received: 26 February 2020; Accepted: 11 March 2020; Published: 26 March 2020

check for updates

\begin{abstract}
Gravitational lensing has been identified as a powerful tool to address fundamental problems in astrophysics at different scales, ranging from exoplanet identification to dark energy and dark matter characterization in cosmology. Image simulations have played a fundamental role in the realization of the full potential of gravitational lensing by providing a means to address needs such as systematic error characterization, pipeline testing, calibration analyses, code validation, and model development. We present a general overview of the generation and applications of image simulations in strong and weak gravitational lensing.
\end{abstract}

Keywords: weak lensing; strong lensing; image simulations; cosmology

\section{Introduction}

Gravitational lensing is defined as the bending of light due to the curvature of space-time caused by any mass-energy distribution, and is explained by the theory of General Relativity (GR) (e.g., [1-3]). Lensing phenomena are diverse and depend on the mass-energy distribution of the lens or deflector (e.g., galaxies, cluster of galaxies, the dark matter large scale structure, etc.), as well as on the relative geometric configuration between the source (e.g., distant galaxies, quasars, or even the cosmic microwave background), the lens, and the observer. It is therefore useful and customary to identify several regimes of lensing depending on the particular configuration of the system at hand $[4,5]$. The case in which multiple images and arcs are produced is known as strong lensing $[6,7]$, whereas the less dramatic — but much more common—case, in which the lensing signal is so subtle that it can only be detected statistically, is known as weak lensing [8-10]. A third regime known as microlensing uses the magnification of the image of a source star when it is aligned with another lensing star along the line of sight. If the lensing star hosts a planet, its lensing effects can also be measured. This technique is used to discover planets at large distances from the Earth [11-13]. In this paper, we focus on image simulations for strong and weak gravitational lensing. The astrophysical applications of gravitational lensing are numerous. In cosmology, it is a central technique to test the current concordance model $(\Lambda \mathrm{CDM}$, a cosmological constant plus cold dark matter) that is supported by several independent lines of evidence (ranging from scales from the cosmic microwave background to galaxies). This successful model, however, calls for the existence of unknown components-dark matter and dark energy - that, together, constitute about 95\% of the contents of the Universe [14-17]. Strong lensing, in particular, is used to study the distribution of dark matter in galaxies and clusters of galaxies, as well as substructures in dark matter halos, and to probe the distribution of total mass (baryonic and non-baryonic) at small scales [18-23]. The number of strong lensing systems and of giant arcs in them can also be used as a cosmological probe, and measurements of time delays of the multiple images of supernovae and quasars can be used to probe the geometry of the universe and its kinematics through cosmography measurements. In general, strong lensing allows for the determination of cosmological parameters such as the Hubble-Lemaître parameter $\left(H_{0}\right)$ (and to study 
the current $H_{0}$ tension, e.g., [24-27]), the dark energy equation of state ( $\left.w\right)$, and the total mass density $\left(\Omega_{m}\right)$, and for testing alternatives to the standard cosmological model and GR [28-32]. Massive clusters also act as cosmic telescopes, and, through strong lensing magnification, allow for the identification and study of distant galaxies from the early universe, black holes, and quasars and active galactic nuclei that otherwise would not be detected with the native instrument resolutions [33-35]. As probe of both the growth of structure and the expansion history of the Universe, weak lensing of the large-scale structure or cosmic shear has been used to investigate the cause of the observed accelerated expansion of the universe (a form of dark energy or models beyond GR) [16,36-42] (The microlensing regime has been used to set constraints on extended dark matter candidates [43] and to identify exoplanets, as mentioned before.)

While lensing is important for many different applications and its theoretical foundations are well understood, measurement of the signal in each regime is subject to several challenges. Multiple-imaging due to strong lensing is a relatively rare phenomenon as it only affects a small fraction of distant sources [44]. The measurements usually also require subarcsecond resolutions, and, depending on the applications, knowledge of the redshifts of the source and the deflector. When redshift information is necessary and it is not possible to obtain it via spectroscopy, photometric redshifts are often used as estimates. However, these are difficult to calculate in crowded fields due to the mixing of light from the foreground object with the background source $[7,45,46]$. Moreover, in some cases, they might prove to be not accurate enough for the applications at hand [47].

Weak gravitational lensing, on the other hand, is fundamentally limited by shape noise-the standard deviation of the intrinsic galaxy shape distribution- requiring imaging a large number of galaxies to statistically reduce it. To address this limitation, several current (e.g., the Kilo Degree Survey, KiDS (http:/ / www.astro-wise.org/projects/KIDS/), the Hyper Suprime Cam survey, HSC (http:/ / www.naoj.org/Projects/HSC/HSCProject.html), and the Dark Energy Survey, DES (http:/ / www.darkenergysurvey.org/)) and future (e.g., the Dark Energy Spectroscopic Instrument (DESI [48]), the Prime Focus Spectrograph (PFS [49]), the Vera C. Rubin Observatory Legacy Survey of Space and Time (LSST) (LSST (https:/ / www.lsst.org / [50]), Euclid (https: / / sci.esa.int/web / euclid) [51], and the Wide Field Infrared Survey Telescope, WFIRST (https:/ / wfirst.gsfc.nasa.gov/) [52]) projects have and are being designed to collect large amounts of data over large fields of view, reaching a point in which systematic errors are comparable or even dominant over statistical errors. Large datasets will also present an opportunity to increase the number of strong lensing systems found (including more complex systems with multiple sources and deflectors).

Image simulations play a central role in addressing the observational and systematic challenges faced during the measurement of strong and weak gravitational lensing. In general, simulations with known inputs are a fundamental tool for important tasks such as software testing and validation, systematic error characterization, requirement validation, model testing and developing, calibration, analysis testing, etc. Depending on the objective at hand and the computational resources available, there is usually a trade-off between realism and efficiency that results in simulations with varying degrees of complexity (e.g., end-to-end simulations vs. simulations where single parameters are changed one at a time).

The structure of this paper is as follows. Section 2 briefly reviews the basic theory of gravitational lensing (for more detailed reviews, see, e.g., [10,39,41,53]). Section 3 provides an overview of image simulations for strong and weak gravitational lensing studies. Simulations of multiple images and giant arcs in strong lensing by galaxy clusters via ray-tracing is explained, as well as how pipelines for strong lensing can be used to simulate observation through any particular instrument. We discuss the use of simulation in assessing lensing inversion codes for mass modeling, and we also point out the increasing use of simulated strong lensing systems as training sets in finding codes that are based on machine learning techniques. We also discuss the role of simulations in redshift distribution estimations, weak lensing systematic errors characterization, and the creation of mock images that 
include gravitational lensing effects in the context of observations from large astronomical surveys. We conclude in Section 4.

\section{Gravitational Lensing Basics}

Photons from background sources travel along null-geodesics in spacetime. When passing a nearby mass density concentration, their trajectories are bent by an amount determined by the deflection angle, $\hat{\vec{\alpha}}$. The deflection angle can be derived by considering the trajectory of a photon in a weakly (assuming that $\Phi / c^{2} \ll 1$ and $\dot{\Phi}=0$, where $\Phi$ can be thought of as the scalar Newtonian potential that obeys Poisson's equation) perturbed Friedmann-Lemaître-Robertson-Walker metric and solving the perturbed geodesic equation. Alternatively, it can be derived by using Fermat's Principle and assuming that light is traveling through a medium with effective refraction index $n=1-2 \Phi / c^{2}$. The deflection angle is thus given by:

$$
\hat{\vec{\alpha}}=\frac{2}{c^{2}} \int \vec{\nabla}_{\perp} \Phi d s
$$

For a point mass-with a gravitational potential given by $\Phi(r)=-G M / r$ - the deflection angle is given by $4 G M / c^{2} b$, where $b$ is known as the impact parameter or the distance of closest approach to the lens or deflector. For most situations of astrophysical interest, the spatial scales of the lens itself are much smaller than the angular diameter distances between the source and the deflector $\left(D_{d s}\right)$ and between the deflector and the observer $\left(D_{d}\right)$. Under these circumstances, it is possible to use the thin lens approximation, where the 3D mass distribution of the deflector is projected to a 2D plane-the lens plane-perpendicular to the line-of-sight and characterized by its surface-mass density (with $\vec{\xi}$ as the vector in the lens plane):

$$
\Sigma(\vec{\xi})=\int \rho(\vec{\xi}, s) d s
$$

The deflection angle at any point $\vec{\xi}$ is given by the sum of the the contributions due to each individual mass element in the plane:

$$
\hat{\vec{\alpha}}=\frac{4 G}{c^{2}} \int \frac{\left(\vec{\xi}-\vec{\xi}^{\prime}\right) \Sigma\left(\vec{\xi}^{\prime}\right)}{\left|\vec{\xi}-\vec{\xi}^{\prime}\right|^{2}} d^{2} \xi^{\prime 2}
$$

The line-of-sight defines the optical axis between the observer, the lens plane, and the source plane. For a source at an angular position $\vec{\beta}$ that emits a ray of light with an impact parameter $\vec{\xi}=\vec{\theta} D_{d}$ on the lens plane, the general mapping between $\vec{\beta}$ and $\vec{\theta}$ is given by the the lens equation:

$$
\vec{\beta}=\vec{\theta}-\frac{D_{d s}}{D_{s}} \hat{\vec{\alpha}}\left(D_{d} \vec{\theta}\right)=\vec{\theta}-\vec{\alpha}(\vec{\theta})
$$

The lens equation is, in general, non-linear and can have multiple solutions (this is formally the strong lensing regime). The second part of Equation (4) defines the reduced deflection angle $\vec{\alpha}$, which can be written as $\vec{\alpha}=\nabla_{\perp}\left(\frac{2 D_{d s}}{D_{s} c^{2}} \int \Phi d s\right)$. The lens equation thus implies that $(\vec{\theta}-\vec{\beta})$ can be expressed as the gradient of a potential $\psi(\vec{\theta})$, known as the lensing potential, that is a scaled projection of the 3D Newtonian potential $\Phi$

$$
\psi(\vec{\theta})=\frac{2 D_{d s}}{D_{d} D_{s}} \int \Phi\left(D_{d} \vec{\theta}, s\right) d s
$$

Defining $\vec{\nabla}_{\theta}=D_{d} \vec{\nabla}_{\perp}$ as the angular gradient, the lens equation can be written as

$$
\vec{\nabla}_{\theta}\left(\frac{1}{2}(\vec{\theta}-\vec{\beta})^{2}-\psi\right)=0
$$


Equation (6) is Fermat's Principle, $\vec{\nabla}_{\theta} t(\vec{\theta}, \vec{\beta})=0$, with the time delay surface $t(\vec{\theta})$ defined as:

$$
t(\vec{\theta}, \vec{\beta})=\frac{1+z_{d}}{c} \frac{D_{d} D_{s}}{D_{d s}}\left(\frac{1}{2}(\vec{\theta}-\vec{\beta})^{2}-\psi\right)
$$

The time delay surface consists of a geometric term and a gravitational time delay term known as Shapiro delay. Multiple images are manifested as the stationary points of the surface, and their arrival time difference will depend on the Hubble-Lemaître constant through the angular diameter distances. For a point mass, the lens equation reads $\beta=\theta-\frac{D_{d s}}{D_{d} D_{s}} \frac{4 G M}{c^{2} \theta}$. When the source and the lens are aligned, the image formed is a ring with an Einstein radius of

$$
\theta_{E}=\sqrt{\frac{4 G M}{c^{2}} \frac{D_{d s}}{D_{d} D_{s}}}
$$

The Laplacian of the lensing potential is proportional to the surface-mass density of the lens:

$$
\vec{\nabla}_{\theta} \psi=\frac{2 \Sigma\left(D_{d} \vec{\theta}\right)}{\Sigma_{c}} \equiv 2 \kappa
$$

The term $\kappa$ is known as the convergence, and the critical surface mass density is defined as $\Sigma_{c} \equiv \frac{c^{2} D_{s}}{4 \pi G D_{d s} D_{d}}$. In the case when the images of the lensed sources are small compared to the spatial scales in which the deflection angle varies considerably, the lens equation can be linearized to obtain local information of the mapping. Its Jacobian is given by by $A_{i j}=\frac{\partial \beta_{i}}{\partial \theta_{j}}=\delta_{i j}-\frac{\partial \alpha_{i}}{\partial \theta_{j}}$, and can be written as

$$
A=\left(\begin{array}{cc}
1-\kappa & 0 \\
0 & 1-\kappa
\end{array}\right)+\left(\begin{array}{cc}
-\gamma_{1} & \gamma_{2} \\
\gamma_{2} & \gamma_{1}
\end{array}\right)=(1-\kappa)\left(\begin{array}{cc}
1-g_{1} & -g_{2} \\
-g_{2} & 1+g_{1}
\end{array}\right)
$$

where the complex shear $\gamma=\gamma_{1}+i \gamma_{2}$ is defined in terms of the derivatives of the lensing potential as $\gamma_{1} \equiv \frac{1}{2}\left(\partial_{1} \partial_{1} \psi-\partial_{2} \partial_{2} \psi\right)$ and $\gamma_{2} \equiv \partial_{1} \partial_{2} \psi$, and the reduced shear $g$ is defined as $\gamma /(1-\kappa)$. The inverse of the Jacobian in Equation (10) is known as the magnification tensor, and its determinant represents the local magnification $\mu$ in the limit of a point source:

$$
\frac{1}{\mu}=\left|\frac{\mathrm{d}^{2} \beta}{\mathrm{d}^{2} \theta}\right|=|\operatorname{det} \mathrm{A}|=\left(1-\kappa^{2}\right)-\gamma^{2}
$$

The curves in the image plane for which the magnification is formally equal to infinity $(\operatorname{det} A=0)$ are known as critical curves. The corresponding curves in the source plane are known as caustics. In practice, the magnification is never infinite and the finite size of extended sources and other optical effects become important [5]. However, if a source lies close to a caustic curve, it will be highly magnified and distorted, producing images such as giant arcs in cluster, for example.

\section{Image Simulations}

\subsection{Lensing by Galaxy Clusters}

The lens equation in Equation (4) is, in general, non-linear, and it will have multiple solutions if the mass of the deflector is large enough and there is a geometrical alignment between the deflector and the background sources. Multiple images and giant arcs can be produced by systems such as galaxy clusters-the largest gravitationally bound objects in the Universe. To generate simulated images that include the effects of lensing by systems such as these, a mass distribution of the deflector must be produced. For this purpose, analytical models such as a single isothermal sphere or an elliptical power law [54] can be used, with the advantage of being computationally fast, but with the limitation of being too idealized. This limitation can be partially overcome by the use of semi-analytical algorithms such 
as MOKA [55], in which each component of the system (e.g., host dark matter halo, central galaxy, and satellites) is modeled analytically but in which information from state-of-the-art numerical simulations is also used.

The use of full N-body and hydro-dynamical simulations is an approach to model cluster lenses, with the lensing effects later incorporated through ray-tracing algorithms. In $\mathrm{N}$-body simulations, a box is filled with $\mathrm{N}$ massive particles that interact only through gravity (e.g., Millennium [56], Millennium-XXL [57], Dark Sky [58], OuterRim [59], and EuclidFlagship [60]). Hydro-dynamical simulations, in turn, attempt to provide a more accurate description of the properties observed in galaxy clusters by also including physical properties such as gas cooling, heating, and feedback, in addition to dark matter (e.g., Illustris [61], EAGLE [62], MUFASA [63], APOSTLE [64], and RomulusC [65]). For a comprehensive review, see Vogelsberger et al. [66].

\subsubsection{Ray Tracing}

Numerical simulations provide models for the distribution of dark matter and gas in clusters $[67,68]$. The lensing properties of dark matter halos generated through numerical simulations can be studied by the use of the ray tracing technique, in which the trajectory of each individual photon is propagated and followed through the system, assuming that the photons emitted from the source are independent [69-75]. A large number of light rays is sent through the mass distribution of the lens, where the deflection of their trajectories is computed and the distorted and magnified images of the background sources are reconstructed. Since each ray is independent, this approach lends itself to computational parallelization.

Lens models using numerical methods provide information on positions and masses, which can be used to calculate the deflection angle of any light ray that intercepts the lens plane at a given normalized, dimensionless position $\vec{x} \equiv \vec{\xi} / \xi_{0}$ (for some characteristic distance $\xi_{0}$ in the lens plane) by summing the contribution from all the possible lens particles in a system with $N$ particles:

$$
\vec{\alpha}(\vec{x})=\sum_{i=1}^{N} m_{i} \frac{\vec{x}-\vec{x}_{i}}{\left|\vec{x}-\vec{x}_{i}\right|^{2}}
$$

The computational time of this direct approach is of the order of $N^{2}$. A more efficient approach projects the lens particle positions to a regular grid of size $M \times M$, and the mass in each cell is calculated by summing the masses of the particles that belong to that particular cell. A bundle of rays is traced through another regular grid that covers the lens plane, and the deflection angle for each ray $(i, j)$ is computed by adding up the contributions from each cell $(k, l)$ in the grid:

$$
\vec{\alpha}_{i j}=\vec{\alpha}\left(\vec{x}_{i j}\right)=\sum_{k=1}^{M} \sum_{l=1}^{M} m_{k l} \frac{\vec{x}_{i j}-\vec{x}_{k l}}{\left|\vec{x}_{i j}-\vec{x}_{k l}\right|^{2}}
$$

Comparing Equation (13) with the definition of convergence, it can be seen that the deflection angle can be written as a convolution of the convergence with the kernel:

$$
\vec{K}(\vec{x}) \equiv \frac{1}{\pi} \frac{\vec{x}}{|\vec{x}|^{2}}
$$

The deflection angle can be obtained by applying the convolution theorem in Fourier space $(\tilde{\vec{\alpha}}(\vec{k})=2 \pi \tilde{\kappa}(\vec{k}) \tilde{\bar{K}}(\vec{k})$, where the tilde indicates the Fourier transform). Working in Fourier space offers speed advantages (provided that specific boundary and periodicity conditions are met), although the deflection angle can also be obtained by means of direct calculations using tree methods $[72,74,76]$. Once the deflection angles are calculated, lensing properties such as shear and magnification can be 
obtained through the Jacobian of the lens equation (Equation (10)). In the case that multiple deflectors are considered, the lens equation can be generalized to include multiple lens planes, $N_{\mathrm{p}}$ :

$$
\vec{\beta}=\vec{\theta}-\sum_{i=0}^{N_{\mathrm{p}}} \frac{D_{i \mathrm{~s}}}{D_{\mathrm{s}}} \hat{\bar{\alpha}}^{i}\left(\vec{\theta}^{i}\right)
$$

Analogously, multiple source planes can also be included to provide a more accurate representation of the system at hand. For example, in the case of an isolated lens such as a galaxy cluster, the source planes are generated to account for the redshift dependence of the geometric factor $D_{\mathrm{ds}} / D_{\mathrm{s}}$ : source objects are divided into a certain number of redshift bins with centers equally spaced in lensing distance, each one defining a source plane.

\subsubsection{Observation Pipelines}

Using the ray-tracing formalism to include lensing effects, a model of a galaxy cluster or multiple clusters, a given a distribution of sources, and information on observational conditions (e.g., bandpass, detector parameters, integration time, etc.), it is possible to construct an observation pipeline (e.g., Skylens $[69,71,72]$ and PICS [73]) that produces simulated images of systems observed through any particular instrument. The pipeline Skylens, for example, generates source galaxies as denoised postage stamps after drawing from the galaxy sample of the Hubble eXtreme Deep Field (HXDF [77]), and implements the capability to use multiple source and lens planes. Lens models are produced through the use of the semi-analytic code MOKA, although any analytical dark matter halo model or numerical simulation can be used as well. Input parameters such as exposure time $\left(t_{\exp }\right)$, the total throughput function $(T(\lambda)$, which includes the quantum efficiency of the detector, the mirror reflectivity, the transmission curve of the filters and the lenses in the optical system, and the total extinction function), sky coordinates, effective telescope diameter $(D)$, detector gain $(g)$, readout and Poisson noise, and pixel scale $(p)$ must be specified by the user in order to prepare a virtual observation through a chosen instrument and telescope, and calculate the measured flux of a galaxy with a given spectral energy distribution. Following the discussion and formulas of Grazian et al. [78] (see also Plazas et al. [79]), the total photon counts on the detector (in analog-to-digital units (ADU) or digital numbers (DN)) from a source with surface brightness $I(\vec{x}, \lambda)\left(\mathrm{erg} \mathrm{s}^{-1} \mathrm{~cm}^{-2} \mathrm{~Hz}^{-1} \operatorname{arcsec}^{-2}\right)$, is given by

$$
\operatorname{ADU}_{\text {total }}(\vec{x})=\frac{n_{\gamma}(\vec{x})+n_{\text {sky }}+n_{\text {dark }}}{g}
$$

where

$$
\begin{aligned}
n_{\gamma}(\vec{x}) & =\frac{\pi D^{2} t_{\exp } p^{2}}{4 h} \int I(\vec{x}, \lambda) \frac{T(\lambda)}{\lambda} \mathrm{d} \lambda \\
n_{\text {sky }} & =\frac{\pi D^{2} t_{\text {exp }} p^{2}}{4 h} \int \frac{T(\lambda) S(\lambda)}{\lambda} \mathrm{d} \lambda
\end{aligned}
$$

are the contributions from the source and the sky, respectively (with $h$ as Planck's constant and $S(\lambda)$ is the sky flux per square arcsec), and $n_{\text {dark }}$ is the dark current. The zero point of the image, in the $\mathrm{AB}$ system, can be calculated as [78]

$$
\mathrm{ZP}=2.5 \log \left(\frac{\pi D^{2} t_{\exp }}{4 h g} \int \frac{T(\lambda)}{\lambda} \mathrm{d} \lambda\right)-48.6
$$

Once the telescope, the detector, and the deflector (or deflectors) have been defined, Skylens accounts for the tidal effect of matter along the line of sight by using Equation (15) and reconstructs the images of the sources. The images are then convolved by the instrumental PSF, and different sources of noise and sky background are added according to he parameters of the simulated observation. Figure 1 
shows an example of an image simulation of the Hubble Space Telescope (HST) Advance Camera for Surveys Wide Field Channel generated by Skylens through the combination of three different filters.

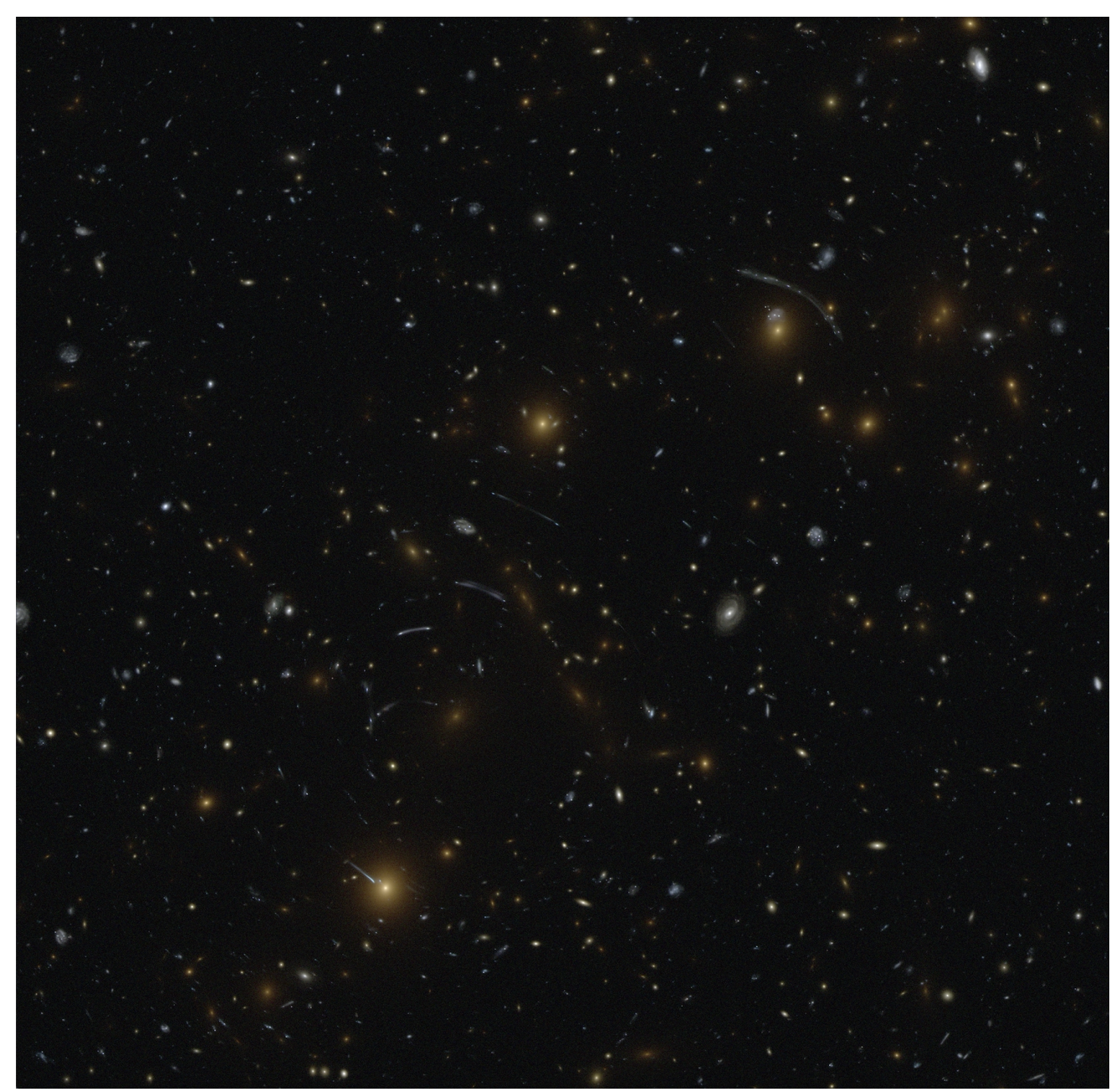

Figure 1. Color composite from images produced by the ray-tracing simulation code Skylens. The image was produced by combining simulated observations taken with the Hubble Space Telescope Advance Camera for Surveys Wide Field Channel in the F475W, F625W, and F775W filters. Taken from Plazas et al. [69].

\subsection{Image Simulations in Weak and Strong Lensing Mass Modeling}

One of the most important applications of strong and weak lensing by galaxy clusters is constraining of the total mass distribution of the lens, dominated by dark matter [80,81]. Strong lensing observables such as flux ratios, relative image positions, and time delays are used in combination with weak lensing, galactic kinematics, and X-ray information to constrain the matter distributions of clusters via a lens inversion process. Knowledge of the matter profile of galaxy clusters is crucial in the understanding of the interplay between dark matter and baryons, as well as the process of large-scale structure formation, and to test the predictions of the standard $\Lambda \mathrm{CDM}$ cosmological model. High-resolution images of galaxy clusters taken with the Hubble Space Telescope ( e.g., CLASH [82] and the HST Frontier Fields $[83,84]$ ) have enabled the community to develop and test different inversion algorithms that, however, do not always result in consistent reconstructions, even when applied to the same systems (see, for example, the analyses of the system MACS J1149.5+2223 by Smith et al. [85] and Zitrin and Broadhurst [86]). The process of mass modeling is subject to many challenges, and, in this context, image simulations provide a valuable tool to assess the performance of inversion codes, to find ways to improve them, and to identify the properties of lenses that are mostly impacted by errors 
during the construction of a lens model (e.g., cluster ellipticities and dynamics, substructures, baryonic physics, etc.). For example, Meneghetti et al. [87] used MOKA and Skylens to simulate strong lensing observations through galaxy clusters with the characteristics of the HST Frontier Fields. The simulations were used to test several lensing inversion methods (parametric, non-parametric or free-form, and hybrid) by different groups.

Simulations with Skylens have also been used to test the robustness of estimating cluster masses by using information from weak lensing, and to study hydrostatic biases by comparing with simulated X-rays observations $[72,76]$.

\subsection{Strong Lensing Simulations and Machine Learning Methods}

Image simulations of strong lensing systems have been used to predict that current and future wide-field galaxy surveys (e.g., DES, HSC survey, KiDS, LSST, and Euclid) will produce several to hundreds of thousands of galaxy-galaxy strong lensing systems [88]. Many efforts have recently focused on employing techniques from computer vision and machine learning to go beyond the traditional approaches (e.g., visual searches of "blue" arcs near "red" galaxies [89], goodness of fit examinations after fitting a model to all candidates $[90,91]$, and public science challenges $[92,93])$ to discover new strong lensing systems in the large datasets, and neural networks have demonstrated to be able to distinguish between simulated lenses and non-lenses [94,95]. Jacobs et al. [96-98] used convolutional neural networks (CNNs, [99]) to produce a catalog of galaxy-galaxy strong lenses (including high-redshift systems) using data from the Dark Energy Survey, and Petrillo et al. [100,101] correspondingly found hundreds of candidates in KiDS data. Jacobs et al. [97] used the LENSPOP [88] code to generate a training set that consists of hundreds of thousands of labeled simulated examples to train a CNN that classifies lenses and non-lenses. Metcalf et al. [102] used N-body (Millenium) and ray-tracing (GLAMER, [74,75]) simulations to analyze a variety of methods including CNN's, visual inspection, and arc finders, to assess their efficiency and completeness, and to identify biases in the face of large future datasets.

\subsection{Image Simulations for Weak Lensing Systematic Errors Characterization}

Recent years have seen the rapid advances in astronomical instrumentation and technology enabling the production of large datasets for astronomical investigations. The decrease in statistical uncertainties in these large datasets results in more stringent requirements to control, characterize, and correct for systematic errors. Weak gravitational lensing of the large-scale structure (cosmic shear) plays a central role in these galaxy surveys as a tool to probe the validity of the standard cosmological model and the nature of the dark sector of the Universe. In turn, the understanding and correction of systematic errors lies at the heart of weak gravitational lensing. Systematic errors include shape measurement precision, PSF measurement and deconvolution, instrument signatures, detector (e.g., charge-couple devices and near-infrared sensors) effects, measurement of photometric redshifts, intrinsic alignments, deblending, etc. (for a comprehensive review, see Mandelbaum [103]). Image simulations with weak gravitational lensing effects have been used to understand these errors and correct for them to the precision required by current and future experiments. End-to-end simulations have been created to bring together the weak lensing community under a common framework and produce challenges such as the Shear TEsting Programme (STEP, [104]) and GRavitational lEnsing Accuracy Testing (GREAT8, [105]). Further iterations of these community-wide challenges (GREAT10 [106] and GREAT3 [107]) focused on simulations in which one parameter a time in the simulation pipeline could be controlled, allowing for a more detailed look at each step in the process of shape galaxy measurement for cosmic shear. The simulations tools to create the images in GREAT3 are publicly available as the modular image simulation code GalSim (https:/ / github.com/GalSim-developers / GalSim) [108], which has been used extensively to study the impact of systematic effects on weak lensing measurements (e.g., [109-114]) or as part of pipelines that create mock images where the selection function of the system is directly measured by inserting simulated objects into real data (e.g., 
Balrog [115] and SynPipe [116]). Simulations have also played a central role in the development, testing, and validation of shear estimation methods (e.g., [117-120]). In the context of the future WFIRST weak lensing program, Troxel et al. [121] used Galsim to render images for a simulation suite designed to carefully study weak lensing systematic errors relevant for WFIRST's High-latitude Imaging Survey [122]. Figure 2 shows examples of images produced with GalSim.

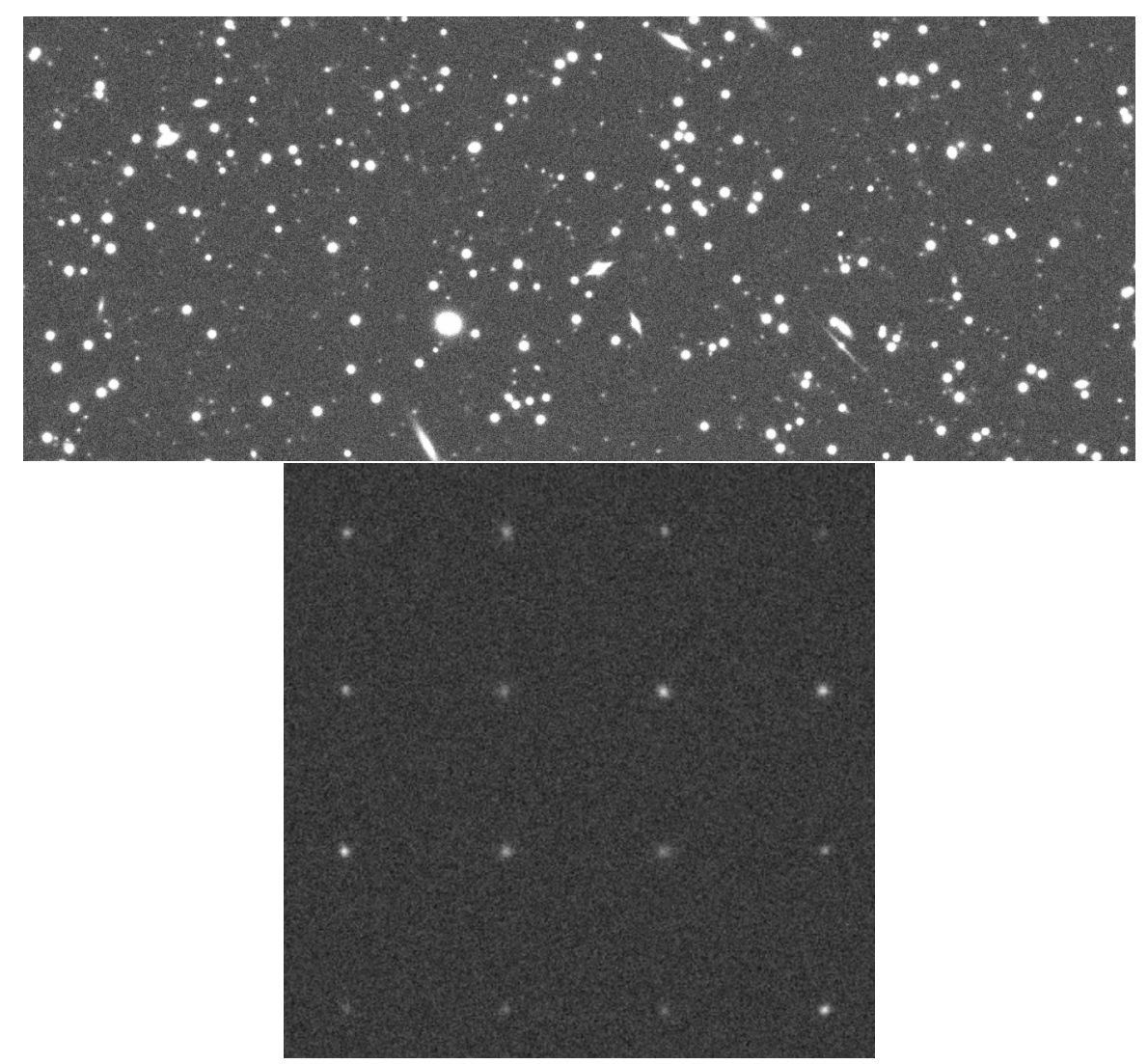

Figure 2. Examples of the types of images that can be generated by GalSim. (Top) Image of a portion of the focal plane of LSST Camera using realistic parameters, including lensing effects. The image was produced with the publicly available configuration file https: / github.com/GalSim-developers / GalSim/blob/master/examples/lsst.yaml. (Bottom) Objects with Gaussian surface brightness profiles arranged in a rectangular grid as well-separated postage stamps.

\subsection{Synthetic Sky Images and Catalogs}

Ray tracing techniques (Section 3.1.1) - in conjunction with full or approximate N-body simulations (e.g., COLA $[123,124]$ ) —are also used in studies of cosmic shear. For example, DeRose et al. [125] used the ADDGALS algorithm to populate dark matter simulations with galaxies, and included gravitational lensing effects via the curved-sky ray-tracing code CALCLENS [126]. They produced a set of 18 synthetic DES Year 1 catalogs to $z=2.35$ and to a depth of $r \approx 26$ that include galaxy properties (e.g., position, ellipticities, and magnitude), photometric errors, and galaxy cluster catalogs (by applying finders such as RedMapper [127]). These simulations have been used to calculate cosmological observables and tests, including quantities relevant for weak gravitational lensing analyses such as correlation functions (galaxy-galaxy, galaxy-shear, and position-position correlation functions; or $3 \times 2$ correlation analyses [16]) and photometric redshift distributions. Simulations such as MICE [128] use a similar approach to DeRose et al. [125], but assume different approximations in the lensing calculation with ray-tracing. Other simulations more focused on weak-lensing statistics using full ray-tracing have also recently been released $[129,130]$. 
Simulated wide-field images are also used to study systematic errors for weak lensing in a more comprehensive framework that includes data to calibrate the images. Bruderer et al. [131] used the Ultra Fast Image Generator (UFIG, Bergé et al. [132]) to produce an implementation of the Monte Carlo Control Loops (MCCL, Refregier and Amara [133]) framework for weak lensing systematic errors studies and applied it to DES data from science verification (e.g., [134]) data. More recently, Kacprzak et al. [135] applied MCCL to DES year 1 data for cosmic shear studies.

Alternative approaches to forward-modeling simulation codes such as UFIG-which might be limited in realism as they require explicit formulation of astrophysical and observational properties-include methodologies that use machine learning methods such as Generative Adversarial Networks (GANs) to let the machine infer both the astrophysical and the instrumentation properties of a dataset [136].

The Dark Energy Science Collaboration of LSST (LSST DESC (https:/ / lsstdesc.org/)) also uses wide-field simulations to prepare for the new challenges that the large and complex dataset produced by the LSST of the Rubin Observatory (approximately 40 billion objects and $50 \mathrm{~Pb}$ of raw data $[137,138]$ ) will generate. The latest of these efforts is referred to as Data Challenge 2 (DC2), the second of three planned synthetic datasets [139] designed to develop and validate data reduction methodologies and to study the impact of systematic effects on LSST data. DC2 includes the production, validation, and analysis of a 5000 sq-deg mock extragalactic catalogs and 300 sq-deg end-to-end simulation. The extragalactic catalog is produced by the CosmoDC2 pipeline [140], and includes the N-body simulation Outer Rim of 1 trillion particles up to $z=10$, produced with the Hybrid/Hardware Accelerated Cosmology Code (HACC) [59]. The simulations include a lensing pipeline that uses particle data from Outer Rim to generate light cones and project the particles in redshift shells and uses a ray-tracing algorithm to produce curved-sky lensing maps. The final available information of objects in the catalogs include positions, sizes, shapes, shear, magnification, convergence, magnitudes, etc. The DC2 image simulations uses a subset of the extragalactic catalogs ( $300 \mathrm{sq}-\mathrm{deg})$ and implements two approaches to produce the images: the Monte Carlo photon shooting code [141] and the code ImSim (https://github.com/LSSTDESC/imSim), which relies on GalSim to produce the images passing specific LSST information.

\subsection{Image Simulations to Assess the Accuracy of Photometric Redshifts}

The determination of accurate galaxy redshift distributions is a key requirement in weak lensing for precision cosmology, especially for the level of requirements needed for the next generation ("stage IV" experiments [142]) galaxy survey projects that will use weak and strong lensing such as LSST, Euclid, and WFIRST [143]. As such, image simulations constitute a valuable tool to test new algorithms and characterize any biases. Bellagamba et al. [144], for example, used Skylens to generate mock images of ground- and space-based surveys in the different bands in the optical and the near-infrared wavelengths and tested the performance of template fitting methods of redshift estimation (e.g., BPZ [145]) under different parameters (such as seeing and depth). Bonnett et al. [146] used simulations created with UFIG to calibrate and validate both template fitting and training methods (where a set of galaxies with known spectroscopic methods is used to train machine-learning algorithms, e.g., TPZ [147], ArborZ [148]) in the context of the DES science verification data.

\section{Conclusions}

We have presented a general overview with some examples of the role that image simulations play in strong and weak gravitational lensing. After 100 years since the formulation of General Relativity, gravitational lensing has positioned itself as one of the most important tools in different areas of astrophysics. In particular, the strong and weak regimes of lensing have become central techniques in current and future experiments that seek to learn more about fundamental problems such as the nature of dark matter and dark energy, the processes of galaxy formation and evolution, the formation of the large-scale structure of the Universe, and the validity of alternative theories of 
gravity. Many efforts are focused on the understanding, characterization, and correction of systematic errors and measurement biases in both regimes. Thanks to these efforts, cosmic shear surveys such as DES have produced the most precise constraints on cosmology from a ground-based survey [16] (with a precision comparable to cosmic microwave background observations), measuring the combination $\sigma_{8}\left(\Omega_{m} / 0.3\right)^{0.5}$ to a precision of about 3.5\% [149]. With the advent of stage IV weak lensing surveys with lower statistical errors such as LSST, WFIRST, and Euclid, the constraints on cosmological parameters will keep improving [150], but only if systematic errors can be controlled. These wide-field surveys will also increase the number of non-transient (e.g., double source plane lenses) and transient (e.g., lensed quasars and supernovae) strong lensing events, which will help place constraints on the dark energy equation of state parameter and on the current $H_{0}$ tension. In this context, the creation of simulated images with known inputs is an invaluable tool to achieve the increased required accuracy demanded by these projects and fulfill their scientific potential.

Funding: This research received no external funding.

Acknowledgments: We thank Massimo Meneghetti for useful discussions and suggestions to improve earlier versions of this manuscript, and Solný A. Aðalsteinsson for proofreading its final version. We thank the Department of Physics of Washington University in St. Louis for their hospitality during the preparation of this paper. This research has made use of NASA's Astrophysics Data System Bibliographic Services. This paper is dedicated to the memory of Colombian astrophysicist Paul D. Nuñez.

Conflicts of Interest: The author declares no conflict of interest.

\section{References}

1. Einstein, A. Kosmologische Betrachtungen zur allgemeinen Relativitätstheorie. In Sitzungsberichte der Königlich Preußischen Akademie der Wissenschaften; Sitzungsberichte der Königlich Preußischen Akademie der Wissenschaften: Berlin, Germany, 1917; pp. 142-152.

2. Misner, C.W.; Thorne, K.S.; Wheeler, J.A. Gravitation; W. H. Freeman: New York, NY, USA, 1973.

3. Carroll, S.M. Spacetime and Geometry. An Introduction to General Relativity; Addison Wesley: San Francisco, CA, USA, 2004.

4. Dodelson, S. Gravitational Lensing; Cambridge University Press: Cambridge, UK, 2017. doi:10.1017/ 9781316424254. [CrossRef]

5. Schneider, P.; Ehlers, J.; Falco, E.E. Gravitational Lenses; Springer: New York, NY, USA, 1992; p. 112. doi:10.1007/978-3-662-03758-4. [CrossRef]

6. Oguri, M. Strong gravitational lensing of explosive transients. arXiv 2019, arXiv:1907.06830, .

7. Treu, T. Strong Lensing by Galaxies. Annu. Rev. Astron. Astrophys. 2010, 48, 87-125. doi:10.1146/annurevastro-081309-130924. [CrossRef]

8. Bartelmann, M.; Maturi, M. Weak gravitational lensing. Scholarpedia 2017, 12, 32440. doi:10.4249/ scholarpedia.32440. [CrossRef]

9. Bartelmann, M.; Schneider, P. Weak gravitational lensing. Phys. Rep. 2001, 340, 291-472. doi:10.1016/S03701573(00)00082-X. [CrossRef]

10. Schneider, P. Weak Gravitational Lensing. arXiv 2005, arXiv:astro-ph/astro-ph/0509252.

11. Tsapras, Y. Microlensing Searches for Exoplanets. Geosciences 2018, 8, 365. doi:10.3390/geosciences8100365. [CrossRef]

12. Mao, S. Astrophysical applications of gravitational microlensing. Res. Astron. Astrophys. 2012, 12, 947-972. doi:10.1088/1674-4527/12/8/005. [CrossRef]

13. Gaudi, B.S. Microlensing Surveys for Exoplanets. Annu. Rev. Astron. Astrophys. 2012, 50, 411-453. doi:10.1146/annurev-astro-081811-125518. [CrossRef]

14. Weinberg, D.H.; Mortonson, M.J.; Eisenstein, D.J.; Hirata, C.; Riess, A.G.; Rozo, E. Observational probes of cosmic acceleration. Phys. Rep. 2013, 530, 87-255. doi:10.1016/j.physrep.2013.05.001. [CrossRef]

15. Huterer, D.; Shafer, D.L. Dark energy two decades after: observables, probes, consistency tests. Rep. Prog. Phys. 2017, 81, 016901. doi:10.1088/1361-6633/aa997e. [CrossRef] 
16. Abbott, T.M.C.; Abdalla, F.B.; Alarcon, A.; Aleksić, J.; Allam, S.; Allen, S.; Amara, A.; Annis, J.; Asorey, J.; Avila, S.; et al. Dark Energy Survey year 1 results: Cosmological constraints from galaxy clustering and weak lensing. Phys. Rev. D 2018, 98, 043526. doi:10.1103/PhysRevD.98.043526. [CrossRef]

17. Planck Collaboration.; Aghanim, N.; Akrami, Y.; Ashdown, M.; Aumont, J.; Baccigalupi, C.; Ballardini, M.; Banday, A.J.; Barreiro, R.B.; Bartolo, N.; et al. Planck 2018 results. VI. Cosmological parameters. arXiv 2018, arXiv:1807.06209,

18. Dalal, N.; Kochanek, C.S. Strong Lensing Constraints on Small-Scale Linear Power. arXiv 2002, arXiv:astro-ph/astro-ph/0202290.

19. Vegetti, S.; Lagattuta, D.J.; McKean, J.P.; Auger, M.W.; Fassnacht, C.D.; Koopmans, L.V.E. Gravitational detection of a low-mass dark satellite galaxy at cosmological distance. Nature 2012, 481, 341-343. doi:10.1038/nature10669. [CrossRef]

20. Nierenberg, A.M.; Treu, T.; Brammer, G.; Peter, A.H.G.; Fassnacht, C.D.; Keeton, C.R.; Kochanek, C.S.; Schmidt, K.B.; Sluse, D.; Wright, S.A. Probing dark matter substructure in the gravitational lens HE 0435-1223 with the WFC3 grism. Mon. Not. R. Astron. Soc. 2017, 471, 2224-2236. doi:10.1093/mnras/stx1400. [CrossRef]

21. Hezaveh, Y.D.; Dalal, N.; Marrone, D.P.; Mao, Y.Y.; Morningstar, W.; Wen, D.; Bland ford, R.D.; Carlstrom, J.E.; Fassnacht, C.D.; Holder, G.P.; et al. Detection of Lensing Substructure Using ALMA Observations of the Dusty Galaxy SDP.81. Astrophys. J. 2016, 823, 37. doi:10.3847/0004-637X/823/1/37. [CrossRef]

22. Gilman, D.; Birrer, S.; Nierenberg, A.; Treu, T.; Du, X.; Benson, A. Warm dark matter chills out: constraints on the halo mass function and the free-streaming length of dark matter with eight quadruple-image strong gravitational lenses. Mon. Not. R. Astron. Soc. 2020, 491, 6077-6101. doi:10.1093/mnras/stz3480. [CrossRef]

23. Hsueh, J.W.; Enzi, W.; Vegetti, S.; Auger, M.W.; Fassnacht, C.D.; Despali, G.; Koopmans, L.V.E.; McKean, J.P. SHARP - VII. New constraints on the dark matter free-streaming properties and substructure abundance from gravitationally lensed quasars. Mon. Not. R. Astron. Soc. 2020, 492, 3047-3059. [CrossRef]

24. Bernal, J.L.; Verde, L.; Riess, A.G. The trouble withH0. J. Cosmol. Astropart. Phys. 2016, $2016,019$. doi:10.1088/1475-7516/2016/10/019. [CrossRef]

25. Pandey, S.; Raveri, M.; Jain, B. A model independent comparison of supernova and strong lensing cosmography: Implications for the Hubble constant tension. arXiv 2019, arXiv:1912.04325.

26. Shajib, A.J.; Birrer, S.; Treu, T.; Agnello, A.; Buckley-Geer, E.J.; Chan, J.H.H.; Christensen, L.; Lemon, C.; Lin, H.; Millon, M.; et al. STRIDES: A 3.9 per cent measurement of the Hubble constant from the strong lens system DES J0408-5354. arXiv 2019, arXiv:1910.06306.

27. Wong, K.C.; Suyu, S.H.; Chen, G.C.F.; Rusu, C.E.; Millon, M.; Sluse, D.; Bonvin, V.; Fassnacht, C.D.; Taubenberger, S.; Auger, M.W.; et al. H0LiCOW XIII. A 2.4\% measurement of $H_{0}$ from lensed quasars: $5.3 \sigma$ tension between early and late-Universe probes. arXiv 2019, arXiv:1907.04869.

28. Jullo, E.; Natarajan, P.; Kneib, J.P.; D’Aloisio, A.; Limousin, M.; Richard, J.; Schimd, C. Cosmological constraints from strong gravitational lensing in clusters of galaxies. Science 2010, 329, 924-927. doi:10.1126/science.1185759. [CrossRef]

29. Magaña, J.; Motta, V.; Cárdenas, V.H.; Verdugo, T.; Jullo, E. A magnified glance into the dark sector: Probing cosmological models with strong lensing in A1689. Astrophys. J. 2015, 813, 69. doi:10.1088/0004-637x/813/1/69. [CrossRef]

30. Caminha, G.B.; Grillo, C.; Rosati, P.; Balestra, I.; Karman, W.; Lombardi, M.; Mercurio, A.; Nonino, M.; Tozzi, P.; Zitrin, A.; et al. CLASH-VLT: A highly precise strong lensing model of the galaxy cluster RXC J2248.7-4431 (Abell S1063) and prospects for cosmography. Astron. Astrophys. 2016, 587, A80. doi:10.1051/0004-6361/201527670. [CrossRef]

31. Acebron, A.; Cibirka, N.; Zitrin, A.; Coe, D.; Agulli, I.; Sharon, K.; Bradač, M.; Frye, B.; Livermore, R.C.; Mahler, G.; et al. RELICS: Strong-lensing Analysis of the Massive Clusters MACS J0308.9+2645 and PLCK G171.9-40.7. Astrophys. J. 2018, 858, 42. doi:10.3847/1538-4357/aabe29. [CrossRef]

32. Grillo, C.; Rosati, P.; Suyu, S.H.; Balestra, I.; Caminha, G.B.; Halkola, A.; Kelly, P.L.; Lombardi, M.; Mercurio, A.; Rodney, S.A.; et al. Measuring the Value of the Hubble Constant "à la Refsdal". Astrophys. J. 2018, 860, 94. doi:10.3847/1538-4357/aac2c9. [CrossRef]

33. Johnson, T.L.; Sharon, K.; Gladders, M.D.; Rigby, J.R.; Bayliss, M.B.; Wuyts, E.; Whitaker, K.E.; Florian, M.; Murray, K.T. Star Formation at $\mathrm{z}=2.481$ in the Lensed Galaxy SDSS J1110 $=6459$. I. Lens Modeling and Source Reconstruction. Astrophys. J. 2017, 843, 78. doi:10.3847/1538-4357/aa7756. [CrossRef] 
34. Johnson, T.L.; Rigby, J.R.; Sharon, K.; Gladders, M.D.; Florian, M.; Bayliss, M.B.; Wuyts, E.; Whitaker, K.E.; Livermore, R.; Murray, K.T. Star Formation at $\mathrm{z}=2.481$ in the Lensed Galaxy SDSS J1110+6459: Star Formation Down to 30 pc Scales. Astrophys. J.l 2017, 843, L21. doi:10.3847/2041-8213/aa7516. [CrossRef]

35. Livermore, R.C.; Finkelstein, S.L.; Lotz, J.M. Directly Observing the Galaxies Likely Responsible for Reionization. Astrophys. J. 2017, 835, 113. doi:10.3847/1538-4357/835/2/113. [CrossRef]

36. Aylor, K.; Joy, M.; Knox, L.; Millea, M.; Raghunathan, S.; Wu, W.L.K. Sounds Discordant: Classical Distance Ladder and $\Lambda$ CDM-based Determinations of the Cosmological Sound Horizon. Astrophys. J. 2019, 874, 4. doi:10.3847/1538-4357/ab0898. [CrossRef]

37. Poulin, V.; Smith, T.L.; Karwal, T.; Kamionkowski, M. Early Dark Energy can Resolve the Hubble Tension. Phys. Rev. Lett. 2019, 122. doi:10.1103/physrevlett.122.221301. [CrossRef] [PubMed]

38. Di Valentino, E.; Melchiorri, A.; Mena, O. Can interacting dark energy solve the H0 tension? Phys. Rev. D 2017, 96. doi:10.1103/physrevd.96.043503. [CrossRef]

39. Kilbinger, M. Cosmology with cosmic shear observations: A review. Rep. Prog. Phys. 2015, 78, 086901. doi:10.1088/0034-4885/78/8/086901. [CrossRef]

40. Munshi, D.; Valageas, P.; Vanwaerbeke, L.; Heavens, A. Cosmology with weak lensing surveys. Phys. Rep. 2008, 462, 67-121. doi:10.1016/j.physrep.2008.02.003. [CrossRef]

41. Hoekstra, H.; Jain, B. Weak Gravitational Lensing and Its Cosmological Applications. Annu. Rev. Nucl. Part. Syst. 2008, 58, 99-123. [CrossRef]

42. Gunn, J.E. On the Propagation of Light in Inhomogeneous Cosmologies. I. Mean Effects. Astrophys. J. 1967, 150, 737. doi:10.1086/149378. [CrossRef]

43. Alcock, C.; Allsman, R.A.; Alves, D.R.; Axelrod, T.S.; Becker, A.C.; Bennett, D.P.; Cook, K.H.; Dalal, N.; Drake, A.J.; Freeman, K.C.; et al. The MACHO Project: Microlensing Results from 5.7 Years of Large Magellanic Cloud Observations. Astrophys. J. 2000, 542, 281-307. doi:10.1086/309512. [CrossRef]

44. Press, W.H.; Gunn, J.E. Method for Detecting a Cosmological Density of Condensed Objects. Astrophys. J. 1973, 185, 397-412. doi:10.1086/152430. [CrossRef]

45. Jouvel, S.; Host, O.; Lahav, O.; Seitz, S.; Molino, A.; Coe, D.; Postman, M.; Moustakas, L.; Benìtez, N.; Rosati, P.; et al. CLASH: Photometric redshifts with 16 HST bands in galaxy cluster fields. Astron. Astrophys. 2014, 562, A86. doi:10.1051/0004-6361/201322419. [CrossRef]

46. Molino, A.; Benítez, N.; Ascaso, B.; Coe, D.; Postman, M.; Jouvel, S.; Host, O.; Lahav, O.; Seitz, S.; Medezinski, E.; et al. CLASH: Accurate photometric redshifts with 14 HST bands in massive galaxy cluster cores. Mon. Not. R. Astron. Soc. 2017, 470,95-113. doi:10.1093/mnras/stx1243. [CrossRef]

47. González, J.D.R.; Sharon, K.; Mahler, G. An Evaluation of 10 Lensing Models of the Frontier Fields Cluster MACS J0416.1-2403. Astrophys. J. 2018, 863, 60. doi:10.3847/1538-4357/aacf8e. [CrossRef]

48. DESI Collaboration.; Aghamousa, A.; Aguilar, J.; Ahlen, S.; Alam, S.; Allen, L.E.; Allende Prieto, C.; Annis, J.; Bailey, S.; Balland, C.; et al. The DESI Experiment Part I: Science,Targeting, and Survey Design. arXiv 2016, arXiv:1611.00036,

49. Takada, M.; Ellis, R.S.; Chiba, M.; Greene, J.E.; Aihara, H.; Arimoto, N.; Bundy, K.; Cohen, J.; Doré, O.; Graves, G.; et al. Extragalactic science, cosmology, and Galactic archaeology with the Subaru Prime Focus Spectrograph. Publ. Astron. Soc. Jpn. 2014, 66, R1. doi:10.1093/pasj/pst019. [CrossRef]

50. Ivezić, Ž.; Kahn, S.M.; Tyson, J.A.; Abel, B.; Acosta, E.; Allsman, R.; Alonso, D.; AlSayyad, Y.; Anderson, S.F.; Andrew, J.; et al. LSST: From Science Drivers to Reference Design and Anticipated Data Products. Astrophys. J. 2019, 873, 111. doi:10.3847/1538-4357/ab042c. [CrossRef]

51. Laureijs, R.; Amiaux, J.; Arduini, S.; Auguères, J.L.; Brinchmann, J.; Cole, R.; Cropper, M.; Dabin, C.; Duvet, L.; Ealet, A.; et al. Euclid Definition Study Report. arXiv 2011, arXiv:1110.3193.

52. Spergel, D.; Gehrels, N.; Baltay, C.; Bennett, D.; Breckinridge, J.; Donahue, M.; Dressler, A.; Gaudi, B.S.; Greene, T.; Guyon, O.; et al. Wide-Field InfrarRed Survey Telescope-Astrophysics Focused Telescope Assets WFIRST-AFTA 2015 Report. arXiv 2015, arXiv:1503.03757.

53. Bartelmann, M. Gravitational lensing. Class. Quantum Gravity 2010, 27, 233001. doi:10.1088/0264-9381/ 27/23/233001. [CrossRef]

54. Tessore, N.; Benton Metcalf, R. The elliptical power law profile lens. Astron. Astrophys. 2015, 580, A79. doi:10.1051/0004-6361/201526773. [CrossRef] 
55. Giocoli, C.; Meneghetti, M.; Bartelmann, M.; Moscardini, L.; Boldrin, M. MOKA: A new tool for strong lensing studies. Mon. Not. R. Astron. Soc. 2012, 421, 3343-3355. doi:10.1111/j.1365-2966.2012.20558.x. [CrossRef]

56. Springel, V.; White, S.D.M.; Jenkins, A.; Frenk, C.S.; Yoshida, N.; Gao, L.; Navarro, J.; Thacker, R.; Croton, D.; Helly, J.; et al. Simulations of the formation, evolution and clustering of galaxies and quasars. Nature 2005, 435, 629-636. doi:10.1038/nature03597. [CrossRef] [PubMed]

57. Angulo, R.E.; Springel, V.; White, S.D.M.; Jenkins, A.; Baugh, C.M.; Frenk, C.S. Scaling relations for galaxy clusters in the Millennium-XXL simulation. Mon. Not. R. Astron. Soc. 2012, 426, 2046-2062. doi:10.1111/j.1365-2966.2012.21830.x. [CrossRef]

58. Skillman, S.W.; Warren, M.S.; Turk, M.J.; Wechsler, R.H.; Holz, D.E.; Sutter, P.M. Dark Sky Simulations: Early Data Release. arXiv 2014, arXiv:astro-ph.CO/1407.2600.

59. Habib, S.; Pope, A.; Finkel, H.; Frontiere, N.; Heitmann, K.; Daniel, D.; Fasel, P.; Morozov, V.; Zagaris, G.; Peterka, T.; et al. HACC: Simulating sky surveys on state-of-the-art supercomputing architectures. New Astron. 2016, 42, 49-65. doi:10.1016/j.newast.2015.06.003. [CrossRef]

60. Potter, D.; Stadel, J.; Teyssier, R. PKDGRAV3: Beyond Trillion Particle Cosmological Simulations for the Next Era of Galaxy Surveys. arXiv 2016, arXiv:astro-ph.IM/1609.08621.

61. Vogelsberger, M.; Genel, S.; Springel, V.; Torrey, P.; Sijacki, D.; Xu, D.; Snyder, G.; Bird, S.; Nelson, D.; Hernquist, L. Properties of galaxies reproduced by a hydrodynamic simulation. Nature 2014, 509, 177-182. doi:10.1038/nature13316. [CrossRef]

62. Schaye, J.; Crain, R.A.; Bower, R.G.; Furlong, M.; Schaller, M.; Theuns, T.; Dalla Vecchia, C.; Frenk, C.S.; McCarthy, I.G.; Helly, J.C.; et al. The EAGLE project: simulating the evolution and assembly of galaxies and their environments. Mon. Not. R. Astron. Soc. 2014, 446, 521-554. doi:10.1093/mnras/stu2058. [CrossRef]

63. Davé, R.; Thompson, R.; Hopkins, P.F. mufasa: Galaxy formation simulations with meshless hydrodynamics. Mon. Not. R. Astron. Soc. 2016, 462, 3265-3284. doi:10.1093/mnras/stw1862. [CrossRef]

64. Sawala, T.; Frenk, C.S.; Fattahi, A.; Navarro, J.F.; Bower, R.G.; Crain, R.A.; Vecchia, C.D.; Furlong, M.; Helly, J.C.; Jenkins, A.; et al. The APOSTLE simulations: solutions to the Local Group's cosmic puzzles. Mon. Not. R. Astron. Soc. 2016, 457, 1931-1943. doi:10.1093/mnras/stw145. [CrossRef]

65. Tremmel, M.; Quinn, T.R.; Ricarte, A.; Babul, A.; Chadayammuri, U.; Natarajan, P.; Nagai, D.; Pontzen, A.; Volonteri, M. Introducingromulusc: a cosmological simulation of a galaxy cluster with an unprecedented resolution. Mon. Not. R. Astron. Soc. 2018, 483, 3336-3362. doi:10.1093/mnras/sty3336. [CrossRef]

66. Vogelsberger, M.; Marinacci, F.; Torrey, P.; Puchwein, E. Cosmological Simulations of Galaxy Formation. arXiv 2019, arXiv:astro-ph.GA/1909.07976.

67. Gardini, A.; Rasia, E.; Mazzotta, P.; Tormen, G.; De Grandi, S.; Moscardini, L. Simulating Chandra observations of galaxy clusters. Mon. Not. R. Astron. Soc. 2004, 351, 505-514. doi:10.1111/j.1365-2966.2004.07800.x. [CrossRef]

68. Rasia, E.; Mazzotta, P.; Borgani, S.; Moscardini, L.; Dolag, K.; Tormen, G.; Diaferio, A.; Murante, G. Mismatch between X-ray and Emission-weighted Temperatures in Galaxy Clusters: Cosmological Implications. Astrophys. J. 2004, 618, L1-L4. doi:10.1086/427554. [CrossRef]

69. Plazas, A.A.; Meneghetti, M.; Maturi, M.; Rhodes, J. Image simulations for gravitational lensing with SKYLENS. Mon. Not. R. Astron. Soc. 2019, 482, 2823-2832. doi:10.1093/mnras/sty2737. [CrossRef]

70. Killedar, M.; Lasky, P.D.; Lewis, G.F.; Fluke, C.J. Gravitational lensing with three-dimensional ray tracing. Mon. Not. R. Astron. Soc. 2011, 420, 155-169. doi:10.1111/j.1365-2966.2011.20023.x. [CrossRef]

71. Meneghetti, M.; Melchior, P.; Grazian, A.; De Lucia, G.; Dolag, K.; Bartelmann, M.; Heymans, C.; Moscardini, L.; Radovich, M. Realistic simulations of gravitational lensing by galaxy clusters: extracting arc parameters from mock DUNE images. Astron. Astrophys. 2008, 482, 403-418. doi:10.1051/0004-6361:20079119. [CrossRef]

72. Meneghetti, M.; Rasia, E.; Merten, J.; Bellagamba, F.; Ettori, S.; Mazzotta, P.; Dolag, K.; Marri, S. Weighing simulated galaxy clusters using lensing and X-ray. Astron. Astrophys. 2010, 514, A93. doi:10.1051/0004-6361/200913222. [CrossRef]

73. Li, N.; Gladders, M.D.; Rangel, E.M.; Florian, M.K.; Bleem, L.E.; Heitmann, K.; Habib, S.; Fasel, P. PICS: Simulations of Strong Gravitational Lensing in Galaxy Clusters. Astrophys. J. 2016, 828, 54. doi:10.3847/0004-637X/828/1/54. [CrossRef]

74. Metcalf, R.B.; Petkova, M. Glamer-I. A code for gravitational lensing simulations with adaptive mesh refinement. Mon. Not. R. Astron. Soc. 2014, 445, 1942-1953. doi:10.1093/mnras/stu1859. [CrossRef] 
75. Petkova, M.; Metcalf, R.B.; Giocoli, C. Glamer-II. Multiple-plane gravitational lensing. Mon. Not. R. Astron. Soc. 2014, 445, 1954-1966. doi:10.1093/mnras/stu1860. [CrossRef]

76. Rasia, E.; Meneghetti, M.; Martino, R.; Borgani, S.; Bonafede, A.; Dolag, K.; Ettori, S.; Fabjan, D.; Giocoli, C.; Mazzotta, P.; et al. Lensing and X-ray mass estimates of clusters (simulations). New J. Phys. 2012, 14, 055018. doi:10.1088/1367-2630/14/5/055018. [CrossRef]

77. Illingworth, G.D.; Magee, D.; Oesch, P.A.; Bouwens, R.J.; Labbé, I.; Stiavelli, M.; van Dokkum, P.G.; Franx, M.; Trenti, M.; Carollo, C.M.; et al. The HST eXtreme Deep Field (XDF): Combining All ACS and WFC3/IR Data on the HUDF Region into the Deepest Field Ever. Astrophys. J. 2013, 209, 6. doi:10.1088/0067-0049/209/1/6. [CrossRef]

78. Grazian, A.; Fontana, A.; De Santis, C.; Gallozzi, S.; Giallongo, E.; Di Pangrazio, F. The Large Binocular Camera Image Simulator. Publ. Astron. Soc. Pac. 2004, 116, 750-761. doi:10.1086/423123. [CrossRef]

79. Plazas, A.A.; Bernstein, G.M.; Sheldon, E.S. On-Sky Measurements of the Transverse Electric Fields' Effects in the Dark Energy Camera CCDs. Publ. Astron. Soc. Pac. 2014, 126, 750-760. doi:10.1086/677682. [CrossRef]

80. Jullo, E.; Kneib, J.P.; Limousin, M.; Elíasdóttir, Á.; Marshall, P.J.; Verdugo, T. A Bayesian approach to strong lensing modelling of galaxy clusters. New J. Phys. 2007, 9, 447-447. doi:10.1088/1367-2630/9/12/447. [CrossRef]

81. Caminha, G.B.; Rosati, P.; Grillo, C.; Rosani, G.; Caputi, K.I.; Meneghetti, M.; Mercurio, A.; Balestra, I.; Bergamini, P.; Biviano, A.; et al. Strong lensing models of eight CLASH clusters from extensive spectroscopy: Accurate total mass reconstructions in the cores. Astron. Astrophys. 2019, 632, A36. doi:10.1051/0004-6361/201935454. [CrossRef]

82. Postman, M.; Coe, D.; Benítez, N.; Bradley, L.; Broadhurst, T.; Donahue, M.; Ford, H.; Graur, O.; Graves, G.; Jouvel, S.; et al. The Cluster Lensing and Supernova Survey with Hubble: An Overview. Astrophys. J. 2012, 199, 25, doi:10.1088/0067-0049/199/2/25. [CrossRef]

83. Lotz, J.M.; Koekemoer, A.; Coe, D.; Grogin, N.; Capak, P.; Mack, J.; Anderson, J.; Avila, R.; Barker, E.A.; Borncamp, D.; et al. The Frontier Fields: Survey Design and Initial Results. Astrophys. J. 2017, 837, 97. doi:10.3847/1538-4357/837/1/97. [CrossRef]

84. Koekemoer, A.M.; Mack, J.; Lotz, J.M.; Anderson, J.; Avila, R.J.; Barker, E.A.; Borncamp, D.; Gunning, H.C.; Hilbert, B.; Khandrika, H.G.; et al. The HST Frontier Fields: High-Level Science Data Products for the First 4 Completed Clusters, and for the Last 2 Clusters Currently in Progress. Am. Astron. Soc. Meet. Abstr. 2016, $228,315.02$.

85. Smith, G.P.; Ebeling, H.; Limousin, M.; Kneib, J.P.; Swinbank, A.M.; Ma, C.J.; Jauzac, M.; Richard, J.; Jullo, E.; Sand , D.J.; et al. Hubble Space Telescope Observations of a Spectacular New Strong-Lensing Galaxy Cluster: MACS J1149.5+2223 at z = 0.544. Astrophys. J.l 2009, 707, L163-L168. doi:10.1088/0004-637X/707/2/L163. [CrossRef]

86. Zitrin, A.; Broadhurst, T. Discovery of the largest known lensed images formed by a critically convergent lensing cluster. Astrophys. J. 2009, 703, L132-L136. doi:10.1088/0004-637x/703/2/1132. [CrossRef]

87. Meneghetti, M.; Natarajan, P.; Coe, D.; Contini, E.; De Lucia, G.; Giocoli, C.; Acebron, A.; Borgani, S.; Bradac, M.; Diego, J.M.; et al. The Frontier Fields lens modelling comparison project. Mon. Not. R. Astron. Soc. 2017, 472,3177-3216. doi:10.1093/mnras/stx2064. [CrossRef]

88. Collett, T.E. The Population of Galaxy-Galaxy Strong Lenses in Forthcoming Optical Imaging Surveys. Astrophys. J. 2015, 811, 20, doi:10.1088/0004-637X/811/1/20. [CrossRef]

89. Diehl, H.T.; Buckley-Geer, E.J.; Lindgren, K.A.; Nord, B.; Gaitsch, H.; Gaitsch, S.; Lin, H.; Allam, S.; Collett, T.E.; Furlanetto, C.; et al. The DES Bright Arcs Survey: Hundreds of Candidate Strongly Lensed Galaxy Systems from the Dark Energy Survey Science Verification and Year 1 Observations. Astrophys. J. 2017, 232, 15. doi:10.3847/1538-4365/aa8667. [CrossRef]

90. Marshall, P.J.; Hogg, D.W.; Moustakas, L.A.; Fassnacht, C.D.; Bradač, M.; Schrabback, T.; Blandford, R.D. Automated detection of galaxy-scale gravitational lenses in high-resolution imaging data. Astrophys. J. 2009, 694, 924-942. doi:10.1088/0004-637x/694/2/924. [CrossRef]

91. Chan, J.H.H.; Suyu, S.H.; Chiueh, T.; More, A.; Marshall, P.J.; Coupon, J.; Oguri, M.; Price, P. Chitah: Strong-gravitational-lens hunter in imaging surveys. Astrophys. J. 2015, 807, 138. doi:10.1088/0004-637x/ 807/2/138. [CrossRef]

92. Marshall, P.J.; Lintott, C.J.; Fletcher, L.N. Ideas for Citizen Science in Astronomy. Annu. Rev. Astron. Astrophys. 2015, 53, 247-278. doi:10.1146/annurev-astro-081913-035959. [CrossRef] 
93. More, A.; Verma, A.; Marshall, P.J.; More, S.; Baeten, E.; Wilcox, J.; Macmillan, C.; Cornen, C.; Kapadia, A.; Parrish, M.; et al. Space Warps-II. New gravitational lens candidates from the CFHTLS discovered through citizen science. Mon. Not. R. Astron. Soc. 2015, 455, 1191-1210. doi:10.1093/mnras/stv1965. [CrossRef]

94. Lanusse, F.; Ma, Q.; Li, N.; Collett, T.E.; Li, C.L.; Ravanbakhsh, S.; Mandelbaum, R.; Póczos, B. CMU DeepLens: Deep learning for automatic image-based galaxy-galaxy strong lens finding. Mon. Not. R. Astron. Soc. 2017, 473, 3895-3906. doi:10.1093/mnras/stx1665. [CrossRef]

95. Hezaveh, Y.D.; Levasseur, L.P.; Marshall, P.J. Fast automated analysis of strong gravitational lenses with convolutional neural networks. Nature 2017, 548, 555-557. doi:10.1038/nature23463. [CrossRef]

96. Jacobs, C.; Collett, T.; Glazebrook, K.; Buckley-Geer, E.; Diehl, H.T.; Lin, H.; McCarthy, C.; Qin, A.K.; Odden, C.; Escudero, M.C.; et al. An Extended Catalog of Galaxy-Galaxy Strong Gravitational Lenses Discovered in DES Using Convolutional Neural Networks. Astrophys. J. Suppl. Ser. 2019, $243,17$. doi:10.3847/1538-4365/ab26b6. [CrossRef]

97. Jacobs, C.; Collett, T.; Glazebrook, K.; McCarthy, C.; Qin, A.K.; Abbott, T.M.C.; Abdalla, F.B.; Annis, J.; Avila, S.; Bechtol, K.; et al. Finding high-redshift strong lenses in DES using convolutional neural networks. Mon. Not. R. Astron. Soc. 2019, 484, 5330-5349. doi:10.1093/mnras/stz272. [CrossRef]

98. Jacobs, C.; Glazebrook, K.; Collett, T.; More, A.; McCarthy, C. Finding strong lenses in CFHTLS using convolutional neural networks. Mon. Not. R. Astron. Soc. 2017, 471, 167-181. doi:10.1093/mnras/stx1492. [CrossRef]

99. LeCun, Y.; Boser, B.; Denker, J.S.; Henderson, D.; Howard, R.E.; Hubbard, W.; Jackel, L.D. Backpropagation Applied to Handwritten Zip Code Recognition. Neural Comput. 1989, 1, 541-551. [CrossRef]

100. Petrillo, C.E.; Tortora, C.; Chatterjee, S.; Vernardos, G.; Koopmans, L.V.E.; Verdoes Kleijn, G.; Napolitano, N.R.; Covone, G.; Schneider, P.; Grado, A.; et al. Finding strong gravitational lenses in the Kilo Degree Survey with Convolutional Neural Networks. Mon. Not. R. Astron. Soc. 2017, 472, 1129-1150. doi:10.1093/mnras/stx2052. [CrossRef]

101. Petrillo, C.E.; Tortora, C.; Vernardos, G.; Koopmans, L.V.E.; Verdoes Kleijn, G.; Bilicki, M.; Napolitano, N.R.; Chatterjee, S.; Covone, G.; Dvornik, A.; et al. LinKS: Discovering galaxy-scale strong lenses in the Kilo-Degree Survey using convolutional neural networks. Mon. Not. R. Astron. Soc. 2019, 484, 3879-3896. doi:10.1093/mnras/stz189. [CrossRef]

102. Metcalf, R.B.; Meneghetti, M.; Avestruz, C.; Bellagamba, F.; Bom, C.R.; Bertin, E.; Cabanac, R.; Courbin, F.; Davies, A.; Decencière, E.; et al. The strong gravitational lens finding challenge. Astron. Astrophys. 2019, 625, A119. doi:10.1051/0004-6361/201832797. [CrossRef]

103. Mandelbaum, R. Weak Lensing for Precision Cosmology. Annu. Rev. Astron. Astrophys. 2018, 56, $393-433$. doi:10.1146/annurev-astro-081817-051928. [CrossRef]

104. Heymans, C.; Van Waerbeke, L.; Bacon, D.; Berge, J.; Bernstein, G.; Bertin, E.; Bridle, S.; Brown, M.L.; Clowe, D.; Dahle, H.; et al. The Shear Testing Programme-I. Weak lensing analysis of simulated ground-based observations. Mon. Not. R. Astron. Soc. 2006, 368, 1323-1339. doi:10.1111/j.1365-2966.2006.10198.x. [CrossRef]

105. Bridle, S.; Balan, S.T.; Bethge, M.; Gentile, M.; Harmeling, S.; Heymans, C.; Hirsch, M.; Hosseini, R.; Jarvis, M.; Kirk, D.; et al. Results of the GREAT08 Challenge: An image analysis competition for cosmological lensing. Mon. Not. R. Astron. Soc. 2010. doi:10.1111/j.1365-2966.2010.16598.x. [CrossRef]

106. Kitching, T.D.; Balan, S.T.; Bridle, S.; Cantale, N.; Courbin, F.; Eifler, T.; Gentile, M.; Gill, M.S.S.; Harmeling, S.; Heymans, C.; et al. Image analysis for cosmology: results from the GREAT10 Galaxy Challenge. Mon. Not. R. Astron. Soc. 2012, 423, 3163-3208. doi:10.1111/j.1365-2966.2012.21095.x. [CrossRef]

107. Mandelbaum, R.; Rowe, B.; Bosch, J.; Chang, C.; Courbin, F.; Gill, M.; Jarvis, M.; Kannawadi, A.; Kacprzak, T.; Lackner, C.; et al. The third gravitational lensing accuracy testing (great3) challenge handbook. Astrophys. J. Suppl. Ser. 2014, 212, 5. doi:10.1088/0067-0049/212/1/5. [CrossRef]

108. Rowe, B.T.P.; Jarvis, M.; Mandelbaum, R.; Bernstein, G.M.; Bosch, J.; Simet, M.; Meyers, J.E.; Kacprzak, T.; Nakajima, R.; Zuntz, J.; et al. GALSIM: The modular galaxy image simulation toolkit. Astron. Comput. 2015, 10, 121-150. doi:10.1016/j.ascom.2015.02.002. [CrossRef]

109. Plazas, A.A.; Shapiro, C.; Kannawadi, A.; Mandelbaum, R.; Rhodes, J.; Smith, R. The Effect of Detector Nonlinearity onWFIRSTPSF Profiles for Weak Gravitational Lensing Measurements. Publ. Astron. Soc. Pac. 2016, 128, 104001. doi:10.1088/1538-3873/128/968/104001. [CrossRef] 
110. Plazas, A.A.; Shapiro, C.; Smith, R.; Huff, E.; Rhodes, J. Laboratory Measurement of the Brighter-fatter Effect in an H2RG Infrared Detector. Publ. Astron. Soc. Pac. 2018, 130, 065004. doi:10.1088/1538-3873/aab820. [CrossRef]

111. Kannawadi, A.; Shapiro, C.A.; Mandelbaum, R.; Hirata, C.M.; Kruk, J.W.; Rhodes, J.D. The Impact of Interpixel Capacitance on WFIRST PSFs. arXiv 2015, arXiv:astro-ph.IM/1512.01570.

112. Gruen, D.; Bernstein, G.M.; Jarvis, M.; Rowe, B.; Vikram, V.; Plazas, A.A.; Seitz, S. Characterization and correction of charge-induced pixel shifts in DECam. J. Instrum. 2015, 10, C5032. doi:10.1088/1748-0221/ 10/05/C05032. [CrossRef]

113. Lin, C.H.; Tan, B.; Mandelbaum, R.; Hirata, C.M. The Impact of Light Polarization Effects on Weak Lensing Systematics. arXiv 2019, arXiv:astro-ph.IM/1910.05063.

114. Kamath, S.; Meyers, J.E.; Burchat, P.R. Shear measurement bias due to spatially varying spectral energy distributions in galaxies. arXiv 2019, arXiv:astro-ph.CO/1907.04459.

115. Suchyta, E.; Huff, E.M.; Aleksić, J.; Melchior, P.; Jouvel, S.; MacCrann, N.; Ross, A.J.; Crocce, M.; Gaztanaga, E.; Honscheid, K.; et al. No galaxy left behind: accurate measurements with the faintest objects in the Dark Energy Survey. Mon. Not. R. Astron. Soc. 2016, 457, 786-808. doi:10.1093/mnras/stv2953. [CrossRef]

116. Huang, S.; Leauthaud, A.; Murata, R.; Bosch, J.; Price, P.; Lupton, R.; Mandelbaum, R.; Lackner, C.; Bickerton, S.; Miyazaki, S.; et al. Characterization and photometric performance of the Hyper Suprime-Cam Software Pipeline. Publ. Astron. Soc. Jpn. 2017, 70. doi:10.1093/pasj/psx126. [CrossRef]

117. Sheldon, E.S.; Becker, M.R.; MacCrann, N.; Jarvis, M. Metadetection: Mitigating Shear-dependent Object Detection Biases with Metacalibration. arXiv 2019, arXiv:1911.02505,

118. Samuroff, S.; Bridle, S.L.; Zuntz, J.; Troxel, M.A.; Gruen, D.; Rollins, R.P.; Bernstein, G.M.; Eifler, T.F.; Huff, E.M.; Kacprzak, T.; et al. Dark Energy Survey Year 1 results: the impact of galaxy neighbours on weak lensing cosmology with IM3SHAPE. Mon. Not. R. Astron. Soc. 2018, 475, 4524-4543. doi:10.1093/mnras/stx3282. [CrossRef]

119. Bernstein, G.M.; Armstrong, R.; Krawiec, C.; March, M.C. An accurate and practical method for inference of weak gravitational lensing from galaxy images. Mon. Not. R. Astron. Soc. 2016, 459, 4467-4484. doi:10.1093/mnras/stw879. [CrossRef]

120. Plazas, A.A. Weak Gravitational Lensing Systematic Errors in the Dark Energy Survey. Ph.D. Thesis, University of Pennsylvania, Philadelphia, PA, USA, 2012.

121. Troxel, M.A.; Long, H.; Hirata, C.M.; Choi, A.; Jarvis, M.; Mandelbaum, R.; Wang, K.; Yamamoto, M.; Hemmati, S.; Capak, P. A Synthetic WFIRST High-Latitude Imaging Survey: Simulation Suite and the Impact of Wavefront Errors on Weak Gravitational Lensing. arXiv 2019, arXiv:astro-ph.IM/1912.09481.

122. Doré, O.; Hirata, C.; Wang, Y.; Weinberg, D.; Baronchelli, I.; Benson, A.; Capak, P.; Choi, A.; Eifler, T.; Hemmati, S.; et al. WFIRST Science Investigation Team "Cosmology with the High Latitude Survey" Annual Report 2017. arXiv 2018, arXiv:astro-ph.CO/1804.03628.

123. Tassev, S.; Zaldarriaga, M.; Eisenstein, D.J. Solving large scale structure in ten easy steps with COLA. J. Cosmol. Astropart. Phys. 2013, 2013, 036. doi:10.1088/1475-7516/2013/06/036. [CrossRef]

124. Izard, A.; Fosalba, P.; Crocce, M. ICE-COLA: fast simulations for weak lensing observables. Mon. Not. R. Astron. Soc. 2017, 473, 3051-3061. doi:10.1093/mnras/stx2544. [CrossRef]

125. DeRose, J.; Wechsler, R.H.; Becker, M.R.; Busha, M.T.; Rykoff, E.S.; MacCrann, N.; Erickson, B.; Evrard, A.E.; Kravtsov, A.; Gruen, D.; et al. The Buzzard Flock: Dark Energy Survey Synthetic Sky Catalogs. arXiv 2019, arXiv:1901.02401.

126. Becker, M.R. CALCLENS: Weak Lensing Simulations for Large-Area Sky Surveys and Second-Order Effects in Cosmic Shear Power Spectra. Ph.D. Thesis, The University of Chicago, Chicago, IL, USA, 2013.

127. Rykoff, E.S.; Rozo, E.; Busha, M.T.; Cunha, C.E.; Finoguenov, A.; Evrard, A.; Hao, J.; Koester, B.P.; Leauthaud, A.; Nord, B.; et al. redMaPPer. I. algorithm and sdss DR8 catalog. Astrophys. J. 2014, 785, 104. doi:10.1088/0004-637x/785/2/104. [CrossRef]

128. Fosalba, P.; Crocce, M.; Gaztañaga, E.; Castander, F.J. The MICE grand challenge lightcone simulation-I. Dark matter clustering. Mon. Not. R. Astron. Soc. 2015, 448, 2987-3000. doi:10.1093/mnras/stv138. [CrossRef]

129. Takahashi, R.; Hamana, T.; Shirasaki, M.; Namikawa, T.; Nishimichi, T.; Osato, K.; Shiroyama, K. Full-sky Gravitational Lensing Simulation for Large-area Galaxy Surveys and Cosmic Microwave Background Experiments. Astrophys. J. 2017, 850, 24. doi:10.3847/1538-4357/aa943d. [CrossRef] 
130. Harnois-Deraps, J.; Pen, U.L.; Iliev, I.T.; Merz, H.; Emberson, J.D.; Desjacques, V. High-performance P3M N-body code: CUBEP3M. Mon. Not. R. Astron. Soc. 2013, 436, 540-559. doi:10.1093/mnras/stt1591. [CrossRef]

131. Bruderer, C.; Chang, C.; Refregier, A.; Amara, A.; Bergé, J.; Gamper, L. Calibrated Ultra Fast Image Simulations for the Dark Energy Survey. Astrophys. J. 2016, 817, 25. doi:10.3847/0004-637X/817/1/25. [CrossRef]

132. Bergé, J.; Gamper, L.; Réfrégier, A.R.; Amara, A. An Ultra Fast Image Generator (UFIG) for wide-field astronomy. Astron. Comput. 2013, 1, 23-32. doi:10.1016/j.ascom.2013.01.001. [CrossRef]

133. Refregier, A.; Amara, A. A way forward for Cosmic Shear: Monte-Carlo Control Loops. Phys. Dark Universe 2014, 3, 1-3. doi:10.1016/j.dark.2014.01.002. [CrossRef]

134. Abbott, T.; Abdalla, F.; Allam, S.; Amara, A.; Annis, J.; Armstrong, R.; Bacon, D.; Banerji, M.; Bauer, A.; Baxter, E.; et al. Cosmology from cosmic shear with Dark Energy Survey Science Verification data. Phys. Rev. D 2016, 94. doi:10.1103/physrevd.94.022001. [CrossRef]

135. Kacprzak, T.; Herbel, J.; Nicola, A.; Sgier, R.; Tarsitano, F.; Bruderer, C.; Amara, A.; Refregier, A.; Bridle, S.L.; Drlica-Wagner, A.; et al. Monte Carlo Control Loops for cosmic shear cosmology with DES Year 1. arXiv 2019, arXiv:1906.01018.

136. Smith, M.J.; Geach, J.E. Generative deep fields: arbitrarily sized, random synthetic astronomical images through deep learning. arXiv 2019, arXiv:1904.10286.

137. Jurić, M.; Kantor, J.; Lim, K.T.; Lupton, R.H.; Dubois-Felsmann, G.; Jenness, T.; Axelrod, T.S.; Aleksić, J.; Allsman, R.A.; AlSayyad, Y.; et al. The LSST Data Management System. arXiv 2015, arXiv:1512.07914.

138. Ivezic, Z.; Tyson, J.A.; Abel, B.; Acosta, E.; Allsman, R.; AlSayyad, Y.; Anderson, S.F.; Andrew, J.; Angel, R.; Angeli, G.; et al. LSST: From Science Drivers to Reference Design and Anticipated Data Products. arXiv 2008, arXiv:0805.2366.

139. Sánchez, F.J.; Walter, C.W.; Awan, H.; Chiang, J.; Daniel, S.F.; Gawiser, E.; Glanzman, T.; Kirkby, D.P.; Mandelbaum, R.; Slosar, A.; et al. The LSST DESC Data Challenge 1: Generation and Analysis of Synthetic Images for Next Generation Surveys. arXiv 2020, arXiv:2001.00941.

140. Korytov, D.; Hearin, A.; Kovacs, E.; Larsen, P.; Rangel, E.; Hollowed, J.; Benson, A.J.; Heitmann, K.; Mao, Y.Y.; Bahmanyar, A.; et al. CosmoDC2: A Synthetic Sky Catalog for Dark Energy Science with LSST. arXiv 2019, arXiv:1907.06530.

141. Peterson, J.R.; Jernigan, J.G.; Kahn, S.M.; Rasmussen, A.P.; Peng, E.; Ahmad, Z.; Bankert, J.; Chang, C.; Claver, C.; Gilmore, D.K.; et al. Simulation of astronomical images from optical survey telescopes using a comprehensive photon monte carlo approach. Astrophys. J. Suppl. Ser. 2015, $218,14$. doi:10.1088/0067-0049/218/1/14. [CrossRef]

142. Albrecht, A.; Bernstein, G.; Cahn, R.; Freedman, W.L.; Hewitt, J.; Hu, W.; Huth, J.; Kamionkowski, M.; Kolb, E.W.; Knox, L.; et al. Report of the Dark Energy Task Force. arXiv 2006, arXiv:astro-ph/0609591.

143. Hoyle, B.; Gruen, D.; Bernstein, G.M.; Rau, M.M.; De Vicente, J.; Hartley, W.G.; Gaztanaga, E.; DeRose, J.; Troxel, M.A.; Davis, C.; et al. Dark Energy Survey Year 1 Results: Redshift distributions of the weak-lensing source galaxies. Mon. Not. R. Astron. Soc. 2018, 478, 592-610. doi:10.1093/mnras/sty957. [CrossRef]

144. Bellagamba, F.; Meneghetti, M.; Moscardini, L.; Bolzonella, M. Accuracy of photometric redshifts for future weak lensing surveys from space. Mon. Not. R. Astron. Soc. 2012, 422, 553-562. doi:10.1111/j.1365-2966.2012.20632.x. [CrossRef]

145. Benítez, N. Bayesian Photometric Redshift Estimation. Astrophys. J. 2000, 536, 571-583. doi:10.1086/308947. [CrossRef]

146. Bonnett, C.; Troxel, M.; Hartley, W.; Amara, A.; Leistedt, B.; Becker, M.; Bernstein, G.; Bridle, S.; Bruderer, C.; Busha, M.; et al. Redshift distributions of galaxies in the Dark Energy Survey Science Verification shear catalogue and implications for weak lensing. Phys. Rev. D 2016, 94. doi:10.1103/physrevd.94.042005. [CrossRef]

147. Carrasco Kind, M.; Brunner, R.J. TPZ: Photometric redshift PDFs and ancillary information by using prediction trees and random forests. Mon. Not. R. Astron. Soc. 2013, 432, 1483-1501. doi:10.1093/mnras/stt574. [CrossRef]

148. Gerdes, D.W.; Sypniewski, A.J.; McKay, T.A.; Hao, J.; Weis, M.R.; Wechsler, R.H.; Busha, M.T. ArborZ: Photometric redshifts using boosted decision trees. Astrophys. J. 2010, 715, 823-832. doi:10.1088/0004-637x/715/2/823. [CrossRef] 
149. Troxel, M.; MacCrann, N.; Zuntz, J.; Eifler, T.; Krause, E.; Dodelson, S.; Gruen, D.; Blazek, J.; Friedrich, O.; Samuroff, S.; et al. Dark Energy Survey Year 1 results: Cosmological constraints from cosmic shear. Phys. Rev. D 2018, 98. doi:10.1103/physrevd.98.043528. [CrossRef]

150. Schaan, E.; Krause, E.; Eifler, T.; Doré, O.; Miyatake, H.; Rhodes, J.; Spergel, D.N. Looking through the same lens: Shear calibration for LSST, Euclid, and WFIRST with stage 4 CMB lensing. Phys. Rev. D 2017, 95. doi:10.1103/physrevd.95.123512. [CrossRef]

(C) 2020 by the author. Licensee MDPI, Basel, Switzerland. This article is an open access article distributed under the terms and conditions of the Creative Commons Attribution (CC BY) license (http://creativecommons.org/licenses/by/4.0/). 